\title{
A moving boundary model of acrosomal elongation*
}

\author{
Alan S. Perelson ${ }^{1}$ and Evangelos A. Coutsias ${ }^{2}$ \\ 1 Theoretical Division, Los Alamos National Laboratory, Los Alamos, NM 87545, USA \\ ${ }^{2}$ Department of Mathematics, University of New Mexico, Albuquerque, NM 87131, USA
}

\begin{abstract}
A sperm penetrates an egg by extending a long, actin-filled tube known as the acrosomal process. This simple example of biomotility is one of the most dramatic. In Thyone, a $90 \mu \mathrm{m}$ process can extend in less than $10 \mathrm{~s}$. Experiments have shown that actin monomers stored in the base of the sperm are transported to the growing tip of the acrosomal process where they add to the ends of the existing filaments.

The force that drives the elongation of the acrosomal process has not yet been identified although the most frequently discussed candidate is the actin polymerization reaction. Developing what we believe are realistic moving boundary models of diffusion limited actin fiber polymerization, we show that actin filament growth occurs too slowly to drive acrosomal elongation. We thus believe that other forces, such as osmotically driven water flow, must play an important role in causing the elongation. We conjecture that actin polymerization merely follows to give the appropriate shape to the growing structure and to stabilize the structure once water flow ceases.
\end{abstract}

Key words: Acrosomal reaction - Actin polymerization - Moving boundary diffusion - Stefan problem - Fertilization

\section{Introduction}

During fertilization the sperm of an echinoderm, such as the sea cucumber Thyone, penetrates the coats of jelly surrounding an egg by rapidly extending an actin-filled tube known as the acrosomal process (Fig. 1). The force that drives the extension of the acrosomal process has not yet been elucidated, but a number of suggestions have appeared in the literature. Tilney et al. (1973), Tilney (1975), and Tilney and Kallenbach (1979) have suggested that the rapid polymerization of actin, known to occur within the acrosome, provides the force to drive the elongation. Oster et al. (1982) have suggested that osmotically generated hydrostatic forces are responsible for the elongation and that the actin polymerization follows in the already elongating tube. In this model the actin ultimately forms a rigid

* Work partially supported by the United States Department of Energy 


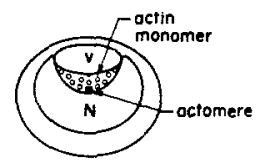

(a)

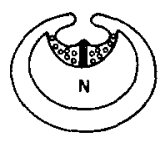

(b)

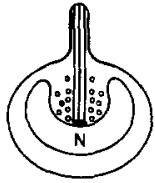

(c)

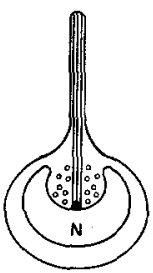

(d)

Fig. 1a-d. The acrosomal reaction. When the sperm comes in contact with the jelly coat surrounding an egg, the acrosomal reaction is initiated. (a) The acrosomal vacuole (v) fuses with the membrane. (b) Actin monomers sequestered in the peracrosomal cup, a region between the nucleus $(N)$ and the vacuole, polymerize onto the actomere. (c) The growing actin filament bundle appears to push into and deform the posterior surface of the vacuole. (d) The vacuole continues to be pushed out, and actin filaments grow

supporting structure that allows the acrosome to keep its shape once equilibrium is established but does not push the acrosomal process outward. In proposing this model, Oster et al. (1982) note that it is difficult to imagine a kinetic process by which a polymerization reaction could push a membrane.

Hill (1981) and Hill and Kirschner (1982) have shown by an equilibrium thermodynamic argument that the free energy change due to actin going from a monomeric to a polymerized state is sufficient to do mechanical work and deform the membrane surrounding an actin filament bundle. So, in principle, the energy released by an actin polymerization reaction could be used by the cell to push the membrane. However, the elongation process is extremely rapid. In Thyone, a $90 \mu \mathrm{m}$ process can extend in somewhat less than $10 \mathrm{~s}$ (Tilney and Inoué 1982). Thus the basic question arises as to whether or not polymerization can occur at this rate within the acrosomal process. If it cannot, then mechanisms such as the osmotic one proposed by Oster et al. need to be studied in more detail. If polymerization can occur at this rate, then one needs to examine the possible mechanisms by which the energy released from the reaction could be used to move the membrane.

In this paper we estimate the maximal rate at which the acrosome could grow if it were driven by polymerization. The polymerization reaction that occurs within the acrosome is of a rather specialized type. Monomer units are stored at the base of the acrosome in a region called the periacrosomal cup (Fig. 1). Within the periacrosomal cup there is a nucleation site for filament growth, known as the actomere (Fig. 1). Once nucleated, filaments grow outward from the actomere by monomer addition (Tilney and Kallenbach 1979). Monomers must thus be transported from the base of the acrosome to the growing tip (Fig. 2). Once a monomer adds to a filament, it elongates the filament. The region of reaction thus moves as the reaction occurs. The polymerization reaction is regulated by actin binding proteins, such as profilin, so as to prevent spontaneous nucleation in the periacrosomal cup and to ensure growth from only one end of the filament (Tilney et al. 1983).

Experiments by Tilney and Inoué $(1982,1985)$ have shown that except for the initial and final stages of growth a graph of $\left(L-L_{0}\right)^{2}$ versus $t$ is linear, where 
Fig. 2. Length of the acrosomal process squared, $L^{2}$, versus time as measured in an experiment by Tilney and Inoué (1982). Note that a straight line fits the data; except during the intial and final stages of the elongation

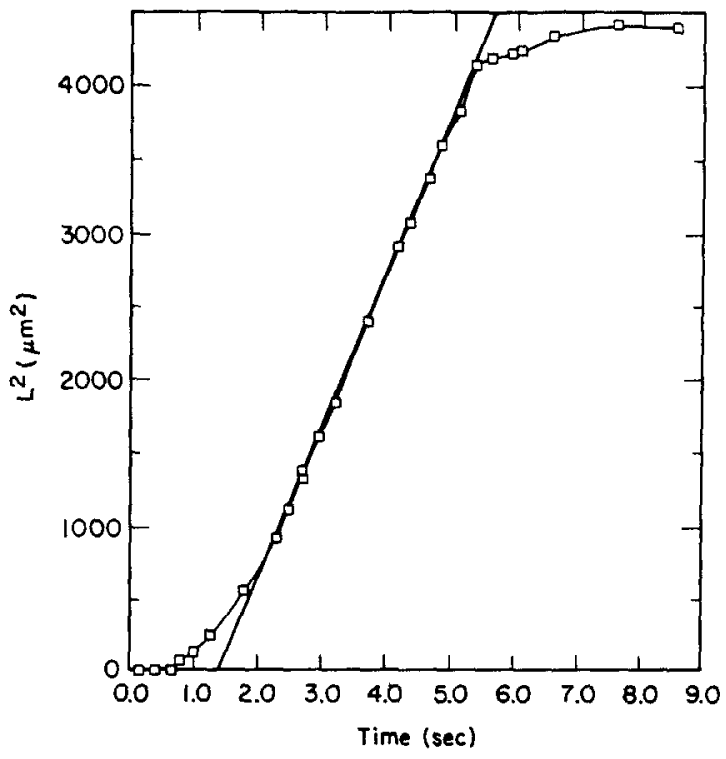

$L-L_{0}$ is the observed length of the acrosomal process (see Fig. 2) and $t$ is time. Because diffusion processes commonly have this behavior, we shall discuss in Sect. 2 a moving boundary diffusion-reaction model for acrosomal extension suggested by Tilney and Kallenbach (1979). In this model, actin polymerization is treated as being irreversible, and thus the reaction behaves as a moving sink for monomer. We correct a minor error in Tilney and Kallenbach's analysis of this model and show that this model gives results in qualitative, but no quantitative, agreement with experimental observation. Thus this model gives graphs of $(L-$ $\left.L_{0}\right)^{2}$ versus $t$ that are linear; however, the final length obtained in $10 \mathrm{sec}$ is significantly smaller than observed.

In Sect. 3 we improve on the model by taking into consideration the convective transport of monomeric actin and by allowing the polymerization reaction to be reversible. Using singular perturbation techniques, we find an approximate solution to the moving boundary reaction-diffusion-convection problem. In Sect. 4 we discuss the relevance of this solution to the biological problem.

Throughout our analysis we shall not be concerned with the initial stages of the process, i.e. vacuole fusion and nucleation of filament growth (see Fig. 1). We assume that at $t=0$, filament growth has begun from a pre-existing filament bundle of length $L_{0}$. Further, we shall ignore any complexities, such as filament capping and cross-linking, introduced by the action of actin binding proteins.

\section{A moving boundary problem for irreversible polymerization}

Tilney and Kallenbach (1979) suggest that the following one-dimensional moving boundary problem might model actin transport and polymerization. A reservoir of monomer at concentration $c_{0}$ serves as a source from which monomers diffuse 
to the right along the $x$-axis. The diffusing monomers are subject to polymerization. Polymerization is modeled as a rapid irreversible absorption into fixed sites distributed uniformly within the volume. Let $s_{0}$ be the total number of sites per unit volume, $c(x, t)$ the concentration of monomer, and $s(x, t)$ the number of free sites per unit volume. Initially there is no monomer in the system, $c(x, 0)=0$, and all sites are free, $s(x, 0)=0$. The dynamics of the system are given by

$$
\begin{aligned}
& \frac{\partial c}{\partial t}=D \frac{\partial^{2} c}{\partial x^{2}}-k c s \\
& \frac{\partial s}{\partial t}=-k c s
\end{aligned}
$$

where $D$ is the monomer diffusion coefficient, and $k$ is a constant that measures the rate of absorption. To convert this to a moving boundary problem that mimics diffusion limited filament growth, let $k \rightarrow \infty$. In this limit, the solution to Eq. (1) is $c s=0$. Thus $s$ will be zero at all points which have been reached by diffusing molecules. Consequently, at any point $x$, either all sites are filled, or none. Freely diffusing monomers will be present only in those volume elements in which all sites are filled. Therefore, there is a moving boundary that separates the filled from the empty region. Let $L(t)$ be the position of the boundary. The rate at which the boundary moves is given by the rate at which sites are filled. Since molecules are supplied by diffusion

$$
-\left.D \frac{\partial c}{\partial x}\right|_{x=L}=s_{0} \frac{d L}{d t},
$$

where the right-hand side, the number of sites occupied per unit time per unit area, is equal to the diffusional flux at the boundary. Because $c=0$ at the boundary, $d c / d t=0$ at $x=L$, and hence

$$
\left.\frac{\partial c}{\partial x}\right|_{x=L} \frac{d L}{d t}+\left.\frac{\partial c}{\partial t}\right|_{x=L}=0 .
$$

Thus, the problem to be solved becomes

$$
\frac{\partial c}{\partial t}=D \frac{\partial^{2} c}{\partial x^{2}}, \quad 0<x<L
$$

with boundary conditions

$$
c(0, t)=c_{0}, \quad t>0
$$

and

initial condition

$$
c(L, t)=0, \quad t>0,
$$

$$
c(x, 0)=0, \quad 0<x<L,
$$

and with the motion of the boundary determined by

$$
\left.D\left(\frac{\partial c}{\partial x}\right)^{2}\right|_{x=L}=\left.s_{0} \frac{\partial c}{\partial t}\right|_{x=L}
$$

Equation (7) arises from solving (3) for $d L / d t$ and substituting into (2). 
This problem is a special case of the one-phase Stefan problem (cf. Cannon 1984). It is well-known that this problem has a unique solution with the boundary moving such that $L(t)$ is proportional to $t^{172}$ (Cannon 1984, pp. 281-288). For this particular version of the Stefan problem, Hermans (1947) shows that

$$
c(x, t)=c_{0}\left\{1-\left[\frac{\operatorname{erf}(x / \sqrt{4 D t})}{\operatorname{erf}(L / \sqrt{4 D t}}\right]\right\}, \quad 0<x<L(t),
$$

where $\operatorname{erf}(\cdot)$ is the error function (Abramowitz and Stegun (1964)), and $L(t) / \sqrt{4 D t}$ is constant. The constant is determined by Eq. (7). Recall that

$$
\frac{d}{d z} \operatorname{erf}(z)=\frac{2}{\sqrt{\pi}} e^{-z^{2}} \text {. }
$$

Using this fact, the chain rule, Eq. (7), and remembering $L / \sqrt{4 D t}$ is constant, we find

$$
\frac{c_{0}}{\sqrt{\pi}} \exp \left(-L^{2} / 4 D t\right)=\frac{s_{0} L}{\sqrt{4 \overline{D t}}} \operatorname{erf}(L / \sqrt{4 D t})
$$

Let $z^{2}=L^{2} / 4 D t$. Then

$$
z \exp \left(z^{2}\right) \operatorname{erf}(z)=\frac{1}{\sqrt{\pi}} \frac{c_{0}}{s_{0}} .
$$

The solution to Eq. (10) provides the required constant, i.e.

$$
L(t)=z \sqrt{4 D t} .
$$

This model can easily be generalized to include a finite supply of molecules from a well-mixed reservoir. Motivated, by the sketch of the biological system in Fig. 3, we consider a reservoir located between $x=-L_{0}$ and $x=0$. Let the monomer concentration in the reservoir be $c_{0}(t)$. Because monomer in the system is supplied by the reservoir, we replace the boundary condition ( $5 \mathrm{a}$ ) by

$$
c(0, t)=c_{0}(t), \quad t>0,
$$

where $c_{0}(t)$ is given by

$$
L_{0}\left(c_{0}(0)-c_{0}(t)\right)=\int_{0}^{L(t)}\left(c+s_{0}\right) d x
$$

Fig. 3. Schematic illustration of monomer transport from the base of the acrosome and of monomer addition to the elongating tips of the actin filaments. The diameter of the growing shaft is greatly exaggerated, as is the space available for monomer transport; typically the diameter of the shaft is one to two percent of that of the base region. In our model the actin filament bundle is assumed to press against the membrane so that the length of the shaft, $L(t)-L_{0}$, is the length of the filament bundle, $L(t)$, minus some initial length $L_{0}$

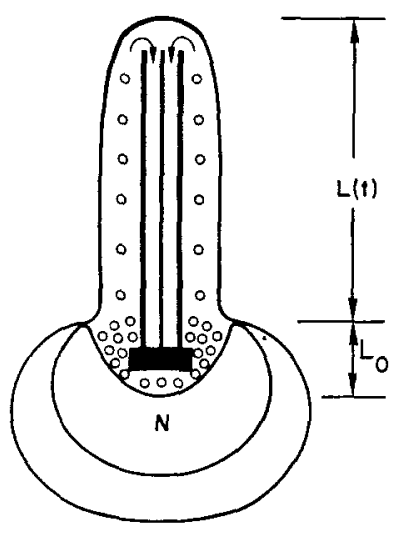


The left side of Eq. (12b) is the amount of monomer supplied by the reservoir up to time $t$, and the right side is the total amount of monomer, free and absorbed, in the system at time $t$. Crank $(1957 \mathrm{a}, \mathrm{b})$ numerically analyzes models of this form. It is easy to see that depletion of the reservoir must slow down the motion of the boundary. We shall not pursue this finite reservoir generalization because, as we show below, even with an infinite reservoir, the motion of the boundary is too slow to model acrosomal growth.

Experiments by Tilney and Inoué (1982) show that if the length of the acrosome squared is plotted against $t$, a straight line results, except for the initial and very final stages of elongation. Thus, to a first approximation the solution to this moving boundary diffusion problem is consistent with data. Tilney and Kallenbach (1979) attempt to examine the rate of growth predicted by Eq. (10) but incorrectly interpret $s_{0}$ as the free concentration of monomer. In what follows we correct their analysis.

Electron micrographs of the acrosomal process show that it is filled with a densely packed bundle of actin filaments containing on average 60 filaments (Tilney and Inoué 1982). Each filament is composed of 370 monomers per micrometer (Aebi et al. 1981). Assuming each monomer occupies one site, the total concentration of "sites" $s_{0}=2.2 \times 10^{7} \mathrm{~cm}^{-1} / \mathrm{A}$, where $A$ is the cross-sectional area of the acrosomal process. Taking the process to be a cylinder with a diameter of $0.05 \mu \mathrm{m}$ (Tilney, personal communication), we find $A=2 \times 10^{-11} \mathrm{~cm}^{2}$ and $s_{0}=1.1 \times 10^{19} \mathrm{~cm}^{-3}$. Tilney and Inoué (1982) estimate that the sperm contains $1.1 \times 10^{6}$ polymerized actin monomers. Let us assume that at the beginning of the acrosomal reaction, the number of monomers is twice this value. This is probably an overestimate since almost all monomers polymerize, and hence we may be overestimating the rate of filament growth by diffusion-limited polymerization. These monomers are stored in the base of the acrosome (periacrosomal cup), a region whose volume is approximately $5 \times 10^{-13} \mathrm{~cm}^{3}$ (Tilney and Inoué 1982). Hence $c_{0}=4.4 \times 10^{18} \mathrm{~cm}^{-3}$. That it is probably an overestimate can be verified from the fact that if one calculates the concentration of actin that one would obtain if the actin molecules were close packed, one finds that $7.87 \times 10^{18}$ actin molecules could fit into $1 \mathrm{~cm}^{3}$ (Tilney and Inoué (1982)). Thus $c_{0}$ is approximately $50 \%$ of the close packed limit.

Using $c_{0}=4.4 \times 10^{18} \mathrm{~cm}^{-3}$ and $s_{0}=1.1 \times 10^{19} \mathrm{~cm}^{-3}$, the right side of Eq. $(10)=$ 0.226 . Solving Eq. (10), we find $z \sim 0.42$. Taking $z=0.42$ and using the value of $D$ given by Tilney and Kallenbach (1979), $D=5 \times 10^{-7} \mathrm{~cm}^{2} / \mathrm{s}$, we find $L^{2} / t=$ $4 D z^{2}=35 \mu \mathrm{m}^{2} / \mathrm{s}$. Hence, in $10 \mathrm{~s}$, with no depletion of monomers from the source, the acrosome would grow $18.7 \mu \mathrm{m}$. This is considerably smaller than the experimental value of $90 \mu \mathrm{m}$.

Tilney and Inoué (1982) measured the slopes of $L^{2}$ versus $t$ plots. In six out of nine sperm, the slopes ranged from $790 \mu \mathrm{m}^{2} / \mathrm{s}$ to $960 \mu \mathrm{m}^{2} / \mathrm{s}$ with a mean of $850 \mu \mathrm{m}^{2} / \mathrm{s}$. The other three sperm produced short processes, and the slopes were about one half of the other six. The slope we predict, $35 \mu \mathrm{m}^{2} / \mathrm{s}$, is approximately 20 -fold smaller than the observed slope.

It thus appears that diffusion limited growth occurs too slowly to be the mechanism driving acrosomal elongation. Tilney and Kallenbach (1979) suggest that convective transport will speed up the transport and allow polymerization 
to drive acrosomal elongation. In the next section we improve our model and examine this conjecture.

\section{A moving boundry problem for reversible polymerization}

The model described by Eqs. (4) - (7) unrealistically treats actin polymerization as a rapid, irreversible reaction. It is known that actin polymerization is reversible. From in vitro experimentation the following kinetic equation has been developed (Wegner 1976):

$$
-\frac{d c}{d t}=\frac{f}{\lambda}\left(k_{1} c-k_{-1}\right)
$$

where for reactions occurring within the acrosomal process we take $f$ to be the number of actin filament ends per unit cross-sectional area and $\lambda$ to be the length a filament extends when a monomer adds to it. Thus $f / \lambda$ is the concentration of filament growing points (ends) in the polymerization zone. If the filament concentration is constant, as will be assumed in our model of the acrosome, $f / \lambda$ may be incorporated into $k_{1}$ and $k_{-1}$.

The values of $k_{1}$ and $k_{-1}$ depend on which end of the actin filament monomers are reacting with. The monomers are asymmetric, and hence the two ends of a filament are distinguishable. Monomers add more rapidly to one end (known as the preferred, or barbed, end) than to the other (known as the nonpreferred, or pointed, end). Tilney and Kallenbach (1979) show that in the acrosomal reaction, growth is from the preferred end. At equilibrium, $c=k_{-1} / k_{1}=\bar{c}$, the "critical" monomer concentration. If $c>\bar{c}$, then filaments tend to grow; whereas if $c<\bar{c}$, filaments tend to shrink. It is thought that $c_{0}>\bar{c}$ so that filaments, once nucleated, grow. Measurements on actin filaments in Limulus sperm (Bonder et al. 1983; Coluccio and Tilney (1984)) suggest that for the preferred end $\bar{c} \cong 10^{-7} \mathrm{M}=$ $6 \times 10^{13} \mathrm{~cm}^{-3}$. We shall assume a similar value is appropriate for Thyone sperm.

To treat the growth of filaments from one end in the presence of reversible reaction requires a model different from that developed in Sect. 2. The end of the filament bundle now corresponds to the boundary, and $L(t)$ is the length of the filament bundle. We assume all filaments have the same length. Thus $L(t)$ can be viewed as the length of a single filament. A filament can only change length when a monomer joins or dissociates from a filament end. The rate of monomer addition per filament end is $-(d c / d t) /(f / \lambda)$. Because a filament extends by length $\lambda$ each time a monomer adds,

$$
\frac{d L}{d t}=\frac{-\lambda^{2}}{f} \frac{d c}{d t}=\lambda\left(k_{1} c(t)-k_{-1}\right),
$$

where Eq. (13) has been substituted for $d c / d t$.

To make this model more realistic, we treat fluid flow within the acrosomal process. As the acrosomal process elongates, it fills with filaments and cytoplasm containing monomeric actin. Viewing the acrosomal process as a pipe of diameter $0.1 \mu \mathrm{m}$ filling with fluid at the speed of the elongation motion $(\sim 10 \mu \mathrm{m} / \mathrm{s})$, one can estimate the Reynolds number as $10^{-6}$. For such low Reynolds number flow 
it seems safe to assume uniform plug flow. The assumption of plug flow was also used in the model of Oster et al. (1982).

The speed at which fluid moves in the acrosome is not known. Passive filling implies fluid motion at the rate of elongation, $\dot{L}$. Faster fluid flows could occur, but they would require a hydrostatic pressure gradient. A pressure gradient would exert a force on the tip and cause the acrosome to extend as in the case of blowing up a balloon (cf. Oster et al. 1982). In order to isolate polymerization as the orily driving force for elongation, we shall restrict or attention to flows that occur at the rate of elongation, $\dot{L}$.

We model the acrosomal process as a cylindrical rod of length $L(t)$. Because of the plug flow assumption, two-dimensional effects inside the rod can be ignored.

Two coordinate systems are useful: the system at rest with origin $x=0$ at the base, and a moving coordinate system $\xi=x-L(t)$ with origin at the tip of the growing process $[\xi=0$ at $x=L(t)]$. In the moving system, the fluid inside the rod is at rest. Initially we use only the rest frame in which $c$, the monomer concentration, satisfies the equation

$$
\frac{\partial c}{\partial t}=D \frac{\partial^{2} c}{\partial x^{2}}-v \frac{\partial c}{\partial x}, \quad 0<x<L(t)
$$

where the fluid velocity $v$, is assumed to be the acrosomal growth rate, i.e.

$$
v=\frac{d L}{d t}
$$

To give the boundary conditions at the two ends, we refer to Fig. 4. At $x=0$, material is entering the rod from the reservoir at speed $d L / d t$. Because the reservoir concentration is $c_{0}$, we assume

$$
c(0, t)=c_{0}, \quad t>0 .
$$

At the tip $x=L(t)$, material enters by diffusion and is added to the growing filament. Thus mass balance and Eq. (14) gives

$$
-\left.D c_{x}\right|_{x=L(t)}=\frac{f}{\lambda} \frac{d L}{d t}=f\left[k_{1} c(L(t), t)-k_{-1}\right]
$$

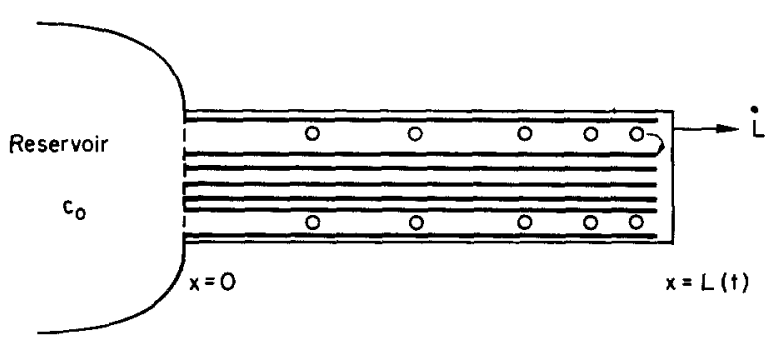

Fig. 4. At the base $(x=0)$ fluid enters containing monomer at the concentration of the reservoir $\left(c=c_{0}\right.$ ). Monomer is transported to the tip by diffusion at rate $-D c_{x}$ and exits the system at rate $f \dot{L} / \lambda$ by addition to the tip 
Convection plays no role in this boundary condition because the convective velocity $v$ exactly matches the tip velocity $\dot{L}$, and hence convection transports no mass to the tip.

Our modeling of acrosomal elongation begins after initiation of the process is completed. At this stage (see Fig. 3)

$$
L(0)=L_{0}
$$

and

$$
c(x, 0)=c_{0}, \quad 0 \leqslant x \leqslant L_{0} .
$$

A limitation of this model is that it assumes a very large reservoir at constant concentration $c_{0}$ that is not depleted by the growth of the filament bundle. Under this assumption growth will go on forever if $c_{0}>\bar{c}=k_{-1} / k_{1}$. In reality, the finite size of the reservoir and the limited amount of extra membrane available to the cell to use to increase its surface area pose effective limits to the final length of the process (Tilney and Inoue 1982). ${ }^{1}$ Still, the conclusions drawn from the model with constant reservoir concentration should be valid at intermediate times and provide a reasonable upper bound on the speed of elongation.

In order to study the effects of reversible polymerization on the dynamics, we shall first rewrite the system (15)-(20) in terms of dimensionless variables. We introduce the dimensionless quantities $\tilde{c}, \tilde{t}, \tilde{x}, \tilde{L}$, etc., by

$$
c=\gamma \tilde{c}, \quad t=\tau \tilde{i}, \quad L=\zeta \tilde{L}, \quad x=\zeta \tilde{x}, \quad \xi=\zeta \tilde{\xi} .
$$

Equation (15) gives

$$
\frac{\partial \tilde{c}}{\partial \tilde{t}}=\left(\frac{D \tau}{\zeta^{2}}\right) \frac{\partial^{2} \tilde{c}}{\partial \tilde{x}^{2}}-\left(\frac{d \tilde{L}}{d \tilde{t}}\right) \frac{\partial \tilde{c}}{\partial \tilde{x}}
$$

and we let

$$
\tilde{D}=D \tau / \zeta^{2}
$$

be the dimensionless diffusion coefficient. Following the discussion in the previous section, we take $D=5 \times 10^{-7} \mathrm{~cm}^{2} / \mathrm{s}$, and we choose as typical length and time scales the values $\zeta=90 \mu \mathrm{m}$ and $\tau=10 \mathrm{~s}$. Thus

$$
\tilde{D}=0.06 \text {. }
$$

The dimensionless versions of the boundary conditions (17)-(18) are

$$
\tilde{c}(0, \tilde{t})=c_{0} / \gamma
$$

${ }^{1}$ If $V$ is the volume of the reservoir, then the $c_{0} V$ monomers, each of length $\lambda$, could be assembled into $f A$ filaments with total length at most $\lambda c_{0} V /(f A)$ 
and

$$
-\left.\tilde{D} \frac{\partial \tilde{c}}{\partial \tilde{x}}\right|_{\tilde{x}=\tilde{L}}=\tilde{f} \frac{d \tilde{L}}{d \tilde{t}}=\tilde{f}\left[\tilde{k}_{1} c_{1}(\tilde{L}, \tilde{t})-\tilde{k}_{-1}\right]
$$

where

$$
\begin{gathered}
\tilde{f}=\frac{f}{\lambda \gamma}, \\
\tilde{k}_{1}=\frac{\tau \lambda \gamma}{\zeta} k_{1},
\end{gathered}
$$

and

$$
\tilde{k}_{-1}=\frac{\tau \lambda}{\zeta} k_{-1}
$$

As typical values, we take

$$
\begin{gathered}
f=60 \text { filaments } / 2 \times 10^{-11} \mathrm{~cm}^{2}=3 \times 10^{12} \mathrm{~cm}^{-2}, \\
\lambda=10^{-4} \mathrm{~cm} / 370 \text { monomers }=2.7 \times 10^{-7} \mathrm{~cm}
\end{gathered}
$$

and

$$
\gamma=c_{0}=4.4 \times 10^{18} \mathrm{~cm}^{-3} .
$$

Thus

$$
\tilde{f}=2.5 \text {. }
$$

It is of interest to note that in terms of the variables used in Sect. 2,

$$
s_{0}=f / \lambda
$$

and thus

$$
\tilde{f}=s_{0} / c_{0} .
$$

From the data of Bonder et al. (1983),

$$
k_{1}=2 \times 10^{-14} \mathrm{~cm}^{3} / \mathrm{s}
$$

and

$$
k_{-1}=2 \mathrm{~s}^{-1} \text {. }
$$

Similar values have been reported by Pollard and Mooseker (1981), i.e. $k_{1}=$ $10^{-14} \mathrm{~cm}^{3} / \mathrm{s}$ and $k_{-1}=6 \mathrm{~s}^{-1}$; and by Coluccio and Tilney (1984), i.e. $k_{1}=$ $0.56 \times 10^{-14} \mathrm{~cm}^{3} / \mathrm{s}$ and $k_{-1}=0.3 \mathrm{~s}^{-1}$. Taking the values in Eq. (31) as being typical, we find

$$
\tilde{k}_{1}=26.4
$$

and

$$
\tilde{k}_{-1}=6 \times 10^{-4}
$$

Finally, we take

$$
\tilde{n}=\tilde{c}-1
$$


In terms of the dimensionless quantities introduced above, the system becomes [here, for simplicity, we drop all the $(\sim)$ signs from our variables]

$$
\begin{gathered}
n_{t}+\dot{L} n_{x}=D n_{x x}, \quad 0<x<L(t) \\
n(x, 0)=0, \quad 0 \leqslant x \leqslant L_{0}, \\
n(0, t)=0,
\end{gathered}
$$

and

$$
-\left.D n_{x}\right|_{x=L(t)}=f\left(k_{1} n+k_{1}-k_{-1}\right) .
$$

An analytical study of the evolution of the moving boundary-initial value problem given by Eq. (34) is very difficult. Because $D \cong 0.06$, we shall pursue a singular perturbation analysis of (34) when $D$ is small. Anticipating a boundary layer of thickness $\sqrt{D}$ at the tip, we introduce

$$
\varepsilon=D^{1 / 2} \approx 0.24
$$

as our perturbation parameter. On studying a problem dependent on a small parameter, $\varepsilon$, by singular perturbation techniques, our aim is to produce an asymptotic expansion for the solution uniformly valid in the domain of interest as $\varepsilon \rightarrow 0$. In practice, the results retain their asymptotic validity (in an approximating sense) for $\varepsilon$ not so small, but the expansion might require more terms to provide a reasonable estimate for the solution (see, e.g. Murray (1984)).

If we let $\varepsilon \rightarrow 0$, Eq. (34), the "outer" problem, has the solution $n(x, t)=0$ [i.e. $c(x, t)=1]$, which fails to satisfy the boundary condition at $x=L(t)$. Observe that in Eq. (34) the term $D n_{x x}$ is negligible (of order $D=\varepsilon^{2}$ ) unless $n_{x x}$ is large (of order $D^{-1}$ or $\varepsilon^{-2}$ ). This will happen in a neighborhood of the tip of thickness $\sqrt{D}$ (Fig. 5). To show this explicitly we employ a stretched coordinate at the tip

$$
z=\frac{\xi}{\varepsilon}=\frac{x-L(t)}{\varepsilon}
$$

which, in standard terminology, is called the "inner" variable. In terms of $z$, the region $0<x<L(t)$ becomes $-L / \varepsilon<z<0$ so that the left end point $\rightarrow-\infty$ as $\varepsilon \rightarrow 0$. In keeping with this, the zeroth order "inner" problem must be solved on a semi-infinite interval and we require a solution that tends to the (constant) reservoir value $c_{0}$ as $z \rightarrow-\infty$. Since in reality our left endpoint is at $z=-L / \varepsilon$, not at $z=-\infty$, keeping only the zeroth order term will introduce an error in our calculation that can be accounted for in a self consistent manner, by higher order terms. This turns out not to be necessary because, as we show, the error is negligible (of order $10^{-9}$ ) throughout the domain of interest.

To begin our formal analysis, we state the inner problem in terms of the "stretched" variable $z$, i.e.

$$
\begin{gathered}
n_{t}=n_{z z}, \quad-\infty<z<0, \\
-\varepsilon n_{z}(0, t)=f\left(k_{1} n(0, t)+k_{1}-k_{-1}\right),
\end{gathered}
$$

with

$$
n(z, 0)=0, \quad-\infty<z<0 .
$$




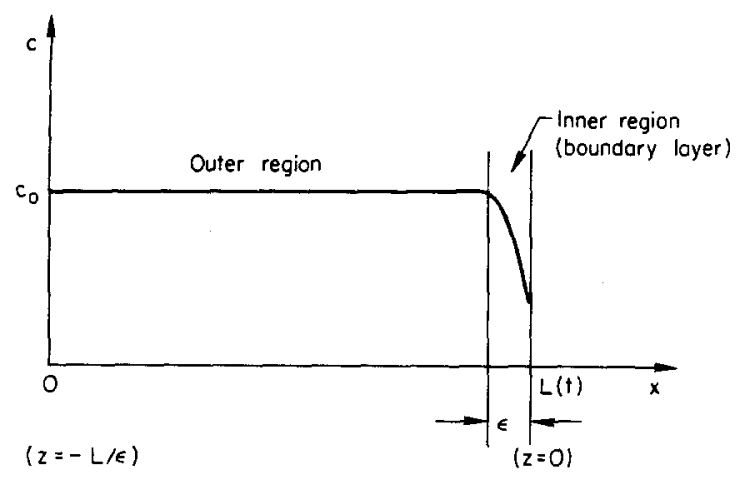

Fig. 5. A schematic illustration of the monomer concentration, $c$, as a function of position, exhibiting the inner (steep growth) and outer (constant) regions

As usual, the solution of the outer problem (Eq. (34)) as $x \rightarrow L$ (i.e. $\xi \rightarrow 0$ ) must be matched to the solution of the inner problem [Eq. (38)] as $z \rightarrow-\infty$ in some sense (cf. Kevorkian and Cole 1979), in order to yield a uniformly valid asymptotic expansion for $n$ (and $c$ ) in the entire interval $0 \leqslant x \leqslant L(t)$. We saw that the solution valid away from the tip is, to leading order $n=0$ or $c=1$. We thus solve the inner problem (Eq. (38)) with the boundary condition

$$
n(-\infty, t)=0
$$

in order to match with the outer solution.

Taking the Laplace transform of Eq. (38), gives

$$
s \hat{n}=\hat{n}_{z z}
$$

and

$$
-\varepsilon \hat{n}_{z}(0, s)=f k_{1} \hat{n}(0, s)+\frac{f\left(k_{1}-k_{-1}\right)}{s}
$$

where the superscript ${ }^{\wedge}$ denotes a Laplace transform, and $s$ is the transform variable. Solving Eq. (40), we find

$$
\hat{n}(z, s)=F(s) e^{z \sqrt{s}}
$$

where the term proportional to $e^{-z \sqrt{s}}$ was neglected because of the boundary condition at $z=-\infty . F(s)$ needs to be determined from the boundary condition (41). Differentiating Eq. (42) and evaluating at $z=0$, gives

$$
-\varepsilon F(s) \sqrt{s}=f k_{1} F(s)+\frac{f\left(k_{1}-k_{-1}\right)}{s},
$$

i.e.

$$
F(s)=\frac{-f\left(k_{1}-k_{-1}\right)}{s\left(f k_{1}+\varepsilon \sqrt{s}\right)}
$$


Thus

$$
\hat{n}(z, s)=\frac{-f\left(k_{1}-k_{-1}\right) e^{z \sqrt{s}}}{s\left(f k_{1}+\varepsilon \sqrt{s}\right)} .
$$

To obtain the inverse Laplace transform of $\hat{n}(z, s)$, we use the following formula (Morse and Feshbach 1953, p. 1582):

$$
\mathbf{L}\left\{\frac{1}{h} \operatorname{erfc}\left(\frac{-z}{2 \sqrt{t}}\right)-\frac{1}{h} e^{-h z+h^{2} t} \operatorname{erfc}\left(\frac{-z}{2 \sqrt{t}}+h \sqrt{t}\right)\right\}=\frac{e^{z \sqrt{s}}}{s(h+\sqrt{s})}
$$

where $\mathbf{L}\{f(t)\}$ denotes the Laplace transform of $f(t)$. For

$$
h=f k_{1} / \varepsilon
$$

the right-hand side of Eq. (45) agrees with Eq. (44) up to a constant, so that

$$
\begin{aligned}
c(z, t) & =1+n(z, t) \\
& =1-\frac{k_{1}-k_{-1}}{k_{1}}\left\{\operatorname{erfc}\left(\frac{-z}{2 \sqrt{t}}\right)-e^{-\left(f k_{1} / \varepsilon\right)\left(z-f k_{1} t / \varepsilon\right)} \operatorname{erfc}\left(\frac{-z}{2 \sqrt{t}}+\frac{f k_{1} \sqrt{t}}{\varepsilon}\right)\right\} .
\end{aligned}
$$

To find $L(t)$, we note

$$
c(z=0, t)=1-\frac{k_{1}-k_{-1}}{k_{1}}\left\{1-e^{\left(f k_{1} / \varepsilon\right)^{2} t} \operatorname{erfc}\left(\frac{f k_{1} \sqrt{t}}{\varepsilon}\right)\right\} .
$$

Since $\dot{L}=k_{1} c(0, t)-k_{-1}$, we find

$$
\dot{L}=\left(k_{1}-k_{-1}\right) e^{\left(f k_{1} / \varepsilon\right)^{2} t} \operatorname{erfc}\left(\frac{f k_{1} \sqrt{t}}{\varepsilon}\right)
$$

which on integration by parts gives

$$
L(t)=L_{0}+\frac{\left(k_{1}-k_{-1}\right) \varepsilon^{2}}{f^{2} k_{1}^{2}}\left[e^{\left(f k_{1} / s\right)^{2} t} \operatorname{erfc}\left(\frac{f k_{1} \sqrt{t}}{\varepsilon}\right)-1+\frac{2 f k_{1} \sqrt{t}}{\varepsilon \sqrt{\pi}}\right] .
$$

Because (Abramowitz and Stegun (1964; p. 298))

$$
\operatorname{erfc}(x) \rightarrow \frac{e^{-x^{2}}}{\sqrt{\pi}}\left(\frac{1}{x}-\frac{1}{2 x^{3}}+\cdots\right), \quad x \gg 1,
$$

we find that for $t=O(1)$ and $\varepsilon$ small

$$
L(t) \cong L_{0}+2\left(\frac{\left(k_{1}-k_{-1}\right) \varepsilon}{f k_{1} \sqrt{\pi}}\right) \sqrt{t}+O\left(\varepsilon^{2}\right) .
$$

Thus after an initial transient the growth is like

$$
\left(L-L_{0}\right)^{2} / t=\text { constant }
$$

in qualitative agreement with experimental data.

Although carrying this perturbation procedure to higher order is unnecessary for our purposes, we comment briefly on what would be involved. We note that Eq. (47), written in terms of the outer variable, is not an exact solution of Eq. 
(34) since it does not satisfy exactly the boundary condition at $x=0$ (i.e. $z=$ $-L / \varepsilon)$; rather,

$$
c\left(\frac{-L}{\varepsilon}, t\right)=1-\frac{k_{1}-k_{-1}}{k_{1}}\left\{\operatorname{erfc}\left(\frac{L}{2 \varepsilon \sqrt{t}}\right)-\exp \left[\frac{f k_{1}\left(L+f k_{1} t\right)}{\varepsilon^{2}}\right] \operatorname{erfc}\left(\frac{1+2 f k_{1} t}{2 \varepsilon \sqrt{t}}\right)\right\} .
$$

For small $\varepsilon$ and $t=O(1)$ we use Eq. (51) and find

$$
c\left(\frac{-L}{\varepsilon}\right) \cong 1-\frac{2\left(k_{1}-k_{-1}\right)}{k_{1}} \sqrt{\frac{t}{\pi}} \varepsilon \exp \left[-\left(\frac{L}{2 \varepsilon \sqrt{t}}\right)^{2}\right]\left(\frac{2 f k_{1} t}{L\left(L+2 f k_{1} t\right)}+O(\varepsilon)\right) .
$$

Since $L / \sqrt{t}$ is constant, the difference between this value and the required value of zero is of order

$$
\varepsilon \exp \left(-\frac{1}{\varepsilon^{2}}\right) \approx 10^{-9}
$$

To get corrections for $c$, we can follow a standard procedure and consider the next order term in a perturbation expansion, i.e.

$$
n_{1}=c-c_{\text {inner }}
$$

Now, $n_{1}$ satisfies the same equations with zero boundary condition at $x=L(t)$ and an $O\left(\varepsilon e^{(-1 / \varepsilon)^{2}}\right)$ boundary condition at $x=0$. We can solve for $n_{1}$ in a similar fashion as we did for $n$ and find the next order correction to our solution. For this it would be necessary to consider corrections on $L(t)$ in a self-consistent manner: we would assume $L=L(t ; \varepsilon)$ and allow for a possible modification of the growth in a slow time scale. Note however that to find $n_{1}$ we solve a diffusion equation with boundary conditions $n_{1}=0$ at $x=L(t)$ and $n_{1} \cong 10^{-9}$ at $x=0$. Clearly, $n_{1}<10^{-9}$ in the domain $0<x<L(t)$ and thus can be neglected when compared with $n$.

Using Eq. (52), we now wish to examine the degree of quantitative agreement with data. Recalling that the quantities in Eq. (52) are all non-dimensional, and that for our typical values, Eq. (32), $\tilde{k}_{-1} \ll \tilde{k}_{1}$, we find

$$
\left(\tilde{L}-\tilde{L}_{0}\right)^{2} / \tilde{t} \cong \frac{4 \tilde{D}}{\tilde{f}^{2} \pi} \text {. }
$$

Expressing $L$ and $t$ in dimensional units, we finally get

$$
\left(L-L_{0}\right)^{2} / t=4 D\left(\frac{c_{0} \lambda}{\sqrt{\pi} f}\right)^{2}=9.9 \mu \mathrm{m}^{2} / \mathrm{s} .
$$

This is 40 - to 80 -fold smaller than the values measured by Tilney and Inoue (1982). It is also smaller than the value we predicted in Sect. 2 using the irreversible absorption model. The reason for this is that the boundary condition used in the absorption model, $c(L, t)=0$, will give rise to a steeper monomer gradient and hence to faster boundary movement than the boundary condition, Eq. (18), used in the diffusion-convection model. 


\section{Discussion}

The elongation of the sperm acrosomal process is one of the most dramatic examples of motility at the cellular level. The speed at which the acrosome grows is in fact comparable to the rate of contraction of some of the fastest skeletal muscles (Tilney and Inoué (1985)). Cytoskeletal rearrangements are intimately involved in cell locomotion, and thus it seemed natural to propose that actin polymerization was involved in generating the forces required to extend the acrosome. Whether or not this is the case has not yet been resolved. The mechanical issue of how an actin polymerization reaction can push a membrane protuberance out from the body of the cell has never been adequately addressed (Oster et al. (1982), Tilney and Inoué (1985)). Further, Oster et al. (1982) and Dan and coworkers $(1964,1967)$ have suggested that osmotic effects may play a major role in extending the acrosomal process.

In order to help evaluate the role of actin polymerization in driving the elongation, we asked whether the kinetics of diffusion-limited actin polymerization were sufficiently rapid to account for the observed acrosomal elongation dynamics. To do this we examined two models. In the first, we assume actin polymerization was infinitely fast and irreversible. The rate of actin filament growth was then determined by solving a moving boundary diffusion problem. In the second, we added convective transport of monomer via plug flow and for completeness incorporated reversible actin polymerization kinetics. Both models predict that a plot of $\left(L-L_{0}\right)^{2}$ versus $t$ should be linear. Thus the models are in qualitative agreement with measurements by Tilney and Inoué $(1982,1985)$. However, when the predicted slope of the $L^{2}$ versus $t$ curves are compared with experimental values, we find that the slopes for both models are 20 to 40 -times too small. Thus, diffusion-limited polymerization can not directly account for the dynamics of acrosomal elongation.

The validity of this conclusion depends upon the realism of our model of actin filament growth. In developing our model we ignored some of the known complexities none of which we believe could substantially alter our conclusions. For example, the actin binding protein profilin is known to play a crucial role. It is thought to work by binding to actin monomer and forming a $1: 1$ complex (Tilney et al. (1983)). Actin monomers are asymmmetric, having two sites: an " $a$ " site at the preferred end and a " $b$ " site at the pointed end. Polymerization occurs by $a$ sites binding to $b$ sites. Profilin regulates the polymerization by binding to and blocking the $a$ site. The actin-profilin complex having only a free $b$ site, can not self-associate, and thus spontaneous filament growth, say in the base of the acrosome, is prevented. The actin-profilin complex having a free $b$ site can bind to an existing filament but only at its preferred end. This partially explains the growth of the filament from one end only. Free actin can also bind to the preferred end, but because much of the actin is in the form of a complex, in the presence of profilin this pathway is of less importance. Thus both free and complexed actin monomers can cause filament elongation. Once the actin-profilin complex binds to a filament, Tilney et al. (1983) believe that a conformational change occurs causing a release of the profilin and the exposure of the $a$ site. The liberated profilin can then bind any free actin, and the process can repeat. Available measurements (Tobacman and Korn (1982), Tseng and Pollard (1982), 
Markey et al. 1982) indicate that the rates of filament elongation from the preferred end in the presence and absence of profilin are surprisingly similar. Thus the reaction kinetics that we have used in the body of the text should be appropriate. However, in the presence of profilin, both free actin and actin-profilin complex must be transported from the base of the acrosome to the growing tip. Profilin has a molecular weight of about 12500 daltons (Tilney et al. 1983), and thus the complex will diffuse somewhat slower than free actin. Thus, including profilin dynamics into a realistic model should further slow down the growth rate of acrosomal elongation. ${ }^{2}$

In addition to profilin, the periacrosomal cup contains three other proteins (Tilney (1979)), 250 000- and 220000-dalton proteins each in 1:12 stoichiometry with actin, and a 25000 dalton protein in a $1: 4$ stoichiometry with actin. The functions of these proteins are not known, but among other things they must be responsible for laterally cross-linking the actin so that the fibers form a bundle. In our model we have ignored the role of actin cross-linking proteins. It is hard to imagine any way that these proteins could speed up the growth process. Although, given their size, they might sterically interfere with the polymerization and slow it down.

If one examines the number of actin filaments as a function of length down the growing acrosomal process, one finds that the largest number of filaments are located near the sperm nucleus, and the fewest are near the tip. Taking transverse sections through the acrosomal process and counting the number of filaments, Tilney and Inoue (1982) show the number changes from 150 near the base to 18 near the tip. The region directly adjoining the tip membrane is difficult to visualize and thus the number 18 is not precise. Further, because of this difficulty in visualization, one cannot tell if the actin filaments touch the membrane as they must if they are to push it. In the model presented in Sect. 3, the change in the number of filaments along the acrosomal process was ignored. Rather we simply used the average number of filaments, 60 . Because the number of filaments decreases, we can presume that capping the ends of some filaments must occur, and that this may be another role for the actin binding proteins found in the periacrosomal cup. To model the observed change in filament density, one could make $f$, the number of filaments per unit cross-sectional area, a function of $x$, the distance down the acrosome, and time and then write a partial differential equation for $f$. We intend to pursue this approach in the future. At present, we can only speculate on the effect of replacing $f$ by $f(x, t)$. Notice from Eq. (14) that $d L / d t$ would not be affected but the boundary conditon, Eq. (18), would. In effect, if some actin filaments are capped, there are less sites available for monomer addition, and thus the monomer concentration, $c$, would decrease more slowly. Because there are more filaments at the base of the acrosome, one would suppose that the monomer would be depleted more than our model predicts

\footnotetext{
2 In models in which the actin concentration in the base is kept constant, the inclusion of profilin will increase the net transport rate of monomers to the tip by a classical facilitated diffusion process. However, if the amount of actin in the base is finite, then including profilin will reduce the concentration of free actin in the base. Transporting a finite amount of actin, partially as a slower moving actin-profilin complex, must necessarily yield a net decrease in overall transport rate
} 
during the early stages of growth, and hence the rate of growth might be somewhat slower than we predict. Conversely, later in the growth process the density of filaments in the reaction zone at the tip would decrease below the constant value of 60 that we used in our model; hence the monomer concentration would be elevated, and the growth dynamics would be enhanced. The net effect on the elongation dynamics is uncertain. One can see from Eq. (56) that the effect of using $f=18$ filaments/unit area, the approximate value found at the distal end, rather than the average value of 60 would only lead to a factor of 3 increase in the slope of the $L^{2}$ versus $t$ plot. If at the tip $f$ were $1-2$ rather than 60 , then the slope would increase by 30-60 and be consistent with experimental observation. However, then only one or two filaments would be available to push the acrosomal membrane, and we doubt enough force could be generated to extend the membrane.

If we are correct and diffusion-plug flow-limited actin polymerization can not occur fast enough to account for the growth of acrosomal process, then what is driving the elongation? Dan and coworkers $(1964,1967)$ and Oster et al. (1982) have suggested that water inflow into the sperm could be responsible. If fluid flow from the base to the tip of the acrosomal process occurs at a rate faster than $d L / d t$, then more monomers will be transported to the tip than we have calculated in our model based on the plug flow assumption. Polymerization can then also occur faster than we have predicted. However, for fluid flow to occur faster than $d L / d t$ a hydrostatic pressure difference between the base and tip is required. This complicates the issue because, as Oster et al. (1982) have shown, water flow driven by a hydrostatic pressure difference can itself drive the elongation and produce a linear $L^{2}$ versus $t$ plot with a slope in agreement with experimental measurements. In the presence of a hydrostatic pressure difference it might be difficult to separate the direct effects of the pressure from the effects of the polymerization, especially if the rate of monomer transport were sufficiently enhanced so as to keep the actin fiber bundle in the vicinity of the tip membrane. If polymerization is not driving the elongation it may play a role in providing directonality to acrosomal growth. Hydrostatic pressure by itself would tend to produce spherical projections such as blebs rather than elongated tubes. Either special membrane asymmetries or effects upon the structure due to the presence of actin filaments must determine the tube-like shape of the acrosomal process. Further, hydrostatic pressures will eventually become equalized and water flows will stop. When this occurs, the actin filaments will stabilize the structure and maintain its tube-like shape.

In the model of Oster et al. the hydrostatic pressure difference is created by osmotically driven water inflow into the periacrosomal cup region of the sperm, an event that occurs within $50-70 \mathrm{~ms}$ of the fusion of the acrosomal vesicle with the plasma membrane (Inoué and Tilney 1982). Recent experiments by Tilney and Inoué (1985) show that changes in the osmotic environment of the sperm affect the dynamics of the elongation process. In a hyperosmotic environment, the slope of the $L^{2}$ versus $t$ curve decreases, whereas in a hypo-osmotic environment, it increases. This is in qualitative agreement with the theory of Oster et al. (1982). Further development of the model is now planned in order to see if quantitative agreement can be obtained between theory and experiment. 
Acknowledgements. We thank James D. Murray, Donald Cohen, and Byron Goldstein for helpful discussions.

\section{References}

1. Abramowitz, M., Stegun, I. A.: Handbook of mathematical functions. Washington, D.C.: National Bureau of Standards 1964

2. Aebi, W., Fowler, W. E., Isenberg, G., Pollard, T. D., Smith, P. R.: Crystalline actin sheets: their structure and polymorphism. J. Cell Biol. 91, 340-351 (1981)

3. Bonder, E. M., Fishkind, D. J., Mooseker, M. S.: Direct measurement of critical concentrations and assembly rate constants at the two ends of an actin filament. Cell 34, 491-501 (1983)

4. Cannon, J. R.: The one-dimensional heat equation, Vol. 23. In: Encyclopeida of mathematics and its applications series. Addison-Wesley, Menlo Park, CA 1984

5. Coluccio, L. M., Tilney, L. G.: Phalloidin enhances assembly by preventing monomer dissociation. J. Cell Biol. 99, 529-535 (1984)

6. Crank, J.: Diffusion with rapid irreversible immobilization. Trans. Faraday Soc. 53, 1083-1091 (1957a)

7. Crank, J.: Two methods for the numerical solution of moving-boundary problems in diffusion and heat flow. Quart. J. Mech. Appl. Math 10 220-231 (1957b)

8. Dan, J. C., Hagiwara, Y.: Studies on the acrosome. IX. Course of acrosome reaction in the starfish. J. Ultrastruct. Res. 18, 562-579. (1967)

9. Dan, J. C., Ohori, Y., Kushida, H.: Studies on the acrosome. VII. Formation of the acrosomal process in sea urchin spermatoza. J. Ultrastruct. Res. 11, 508-524 (1964)

10. Hermans, J. J.: Diffusion with discontinuous boundary. J. Colloid Sci. 2, 387-398 (1947)

11. Hill, T. L.: Microfilament or microtubule assembly or disassembly against a force. Proc. Natl. Acad. Sci. USA 78, 5613-5617 (1981)

12. Hill, T. L., Kirschner, M. W.: Subunit treadmilling of microtubules or actin in the presence of cellular barriers: possible conversion of chemical free energy into mechanical work. Proc. Natl. Acad. Sci. USA 79, 490-494 (1982)

13. Inoué, S., Tilney, L. G.: The acrosomal reaction of Thyone sperm. I. Changes in the sperm head visualized by high resolution video microscopy. J. Cell Biol. 93, 812-819 (1982)

14. Kevorkian, J., Cole, J. D.: Perturbation Methods in Applied Mathematics. Springer, Berlin Heidelberg New York 1981

15. Markey, F., Larsson, H., Weber, K., Lindberg, U.: Nucleation of actin polymerization from profilactin opposite effects of different nuclei. Biochem. Biophys. Acta 704, 43-51 (1982)

16. Morse, P. M., Feshbach, H.: Methods of Theoretical Physics, Part II. McGraw-Hill, New York 1953

17. Murray, J. D.: Asymptotic Analysis. Springer, Berlin Heidelberg New York 1984

18. Oster, G. F., Perelson, A. S., Tilney, L. G.: A mechanical model for elongation of the acrosomal process in Thyone sperm. J. Math. Biol. 15, 259-265 (1982)

19. Pollard, T. D., Mooseker, M. S.: Direct measurement of actin polymerization rate constants by elecron microsopy of actin filaments nucleated by isolated microvillus cores. J. Cell Biol. 88, 654-659 (1981)

20. Tilney, L. G.: The role of actin in nonmuscle cell motility. In: Inoué, S., Stephens, R. E. (eds.) Molecules and cell movement, pp. 339-386. Raven Press, New York 1975

21. Tilney, L. G.: Actin, motility and membranes: In: Cone, R. A., Dowling, J. E. (eds.) Membrane transduction mechanisms, pp. 163-186. Raven Press, New York 1979

22. Tilney, L. G., Bonder, E. M., Coluccio, L. M., Mooseker, M. S.: Actin from Thyone sperm assembles on only one end of an actin filament: a behavior regulated by profilin. J. Cell Biol. 97, 112-124 (1983)

23. Tilney, L. G., Hatano, S., Ishikawa, H., Mooseker, M. S.: The polymerization of actin: its role in the generation of the acrosomal process of certain echinoderm sperm. J. Cell Biol. 59, 109-126 (1973)

24. Tilney, L. G., Kallenbach, N.: Polymerization of actin. VI. The polarity of the actin filaments in the acrosomal process and how it might be determined. J. Cell Biol. 81, 608-623 (1979) 
25. Tilney, L. G., Inoué, S.: Acrosomal reaction of Thyone sperm. II. The kinetics and possible mechanism of acrosomal process elongation. J. Cell Biol. 93, 820-827 (1982)

26. Tobacman, L. S., Korn E. D.: The regulation of actin polymerization and the inhibition of monomeric actin ATPase activity by Acanthamoeba profilin. J. Biol. Chem. 257, 4166-4170 (1982)

27. Tseng, P. C.-H., Pollard, T. D.: Mechanism of a tin of Acanthamoeba profilin: demonstration of actin species specificity regulation by micromolar concentrations of $\mathrm{MgCl}_{2}$. J. Cell Biol. 94, 213-218 (1982)

28. Wegner, A.: Head to tail polymerization of actin. J. Mol. Biol. 108, 139-150 (1976)

Received June 24/Revised July 29, 1985 